\title{
Una radiografía de la industria de la comunicación en Costa Rica. Encuesta a 100 empresas del sector (2016)
}

\section{A Radiography of the Communication Industry in Costa Rica}

\author{
Carolina Carazo Barrantes ${ }^{1}$ \\ Paula Halabi García ${ }^{2}$
}

Fecha de recepción: 13 de febrero de 2018

Fecha de aceptación: 17 de julio de 2018

\begin{abstract}
Resumen
Este artículo presenta un análisis general del quehacer comunicativo en Costa Rica y pretende ser una forma de acercarse a un mejor entendimiento del futuro de esta área y la profesión. El estudio, una encuesta a 100 empresas del sector de la comunicación, da pistas sobre cómo está constituida esta industria en el país (tamaños de las empresas, alcance geográfico, cantidad y perfil de colaboradores, entre otros) y cuáles son sus aportes a la economía nacional (facturación, encadenamientos y trabajo pro-bono). Por tratarse de un estudio exploratorio, se espera que los hallazgos estimulen la discusión en la industria y en la comunidad académica y que esto resulte en la generación de futuras investigaciones de mayor profundidad.

Palabras claves: Agencias digitales, Agencias de publicidad, Agencias de relaciones públicas, Costa Rica, Empresas de comunicación, Mapeo del sector comunicación
\end{abstract}

\begin{abstract}
This article presents a general analysis of the communication industry in Costa Rica and aims to be a way to approach a better understanding of the future of this area and the profession. The study, a survey of 100 companies in the sector, gives clues about how the communication industry is constituted in the country (sizes of companies, geographic scope, number and profile of collaborators, among others) and what its contributions to the national economy are (billing, out-sourcing and pro-bono work). As this is an exploratory study, it is expected that the findings will stimulate discussion in the industry and in the academic community and that this will result in the generation of future studies of greater depth.

KeyWords: Advertising agencies, Communication businesses, Costa Rica, Digital agencies, Mapping of communication sector, Public Relations agencies
\end{abstract}

1. Universidad de Costa Rica, Costa Rica, Investigadora del CICOM, carolina.carazo@ucr.ac.cr

2. Universidad de Costa Rica, Costa Rica, Investigadora de la Escuela de Ciencias de la Comunicación Colectiva, paula.halabi@ucr.ac.cr

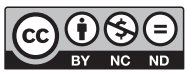




\section{Introducción}

La industria de la comunicación es un sector estratégico en la economía costarricense. Desde hace 30 años el país inició una transformación de una riqueza basada en factores productivos básicos hacia una economía basada en la eficiencia. En esta etapa la economía está sobre la base de la manufactura y sobre el sector de los servicios (Lebendiker, 2014, pp.12-13), en donde la industria de la comunicación ha ido desarrollando una serie de valores agregados y ventajas competitivas que le han permitido crecer, reinventarse y volverse significativa.

¿Y por qué se dice que la comunicación es un sector estratégico? Según Michael Porter (2007), quien define lo estratégico como lo que va a proveer de una ventaja competitiva al mercado, es claro que la industria de la comunicación ha desarrollado un valor para las marcas, las cuales también se han visto beneficiadas por una industria que cuenta con más herramientas para posicionarlas en el mercado y que cada día invierte más en nuevos recursos tecnológicos para trasladar a las marcas esa ventaja competitiva.

Como muchas otras, la industria de la comunicación ha vivido un proceso de transformación en los últimos años. La industria inició el camino de la transición digital hace relativamente poco tiempo, algunos se adelantaron a la ola, pero eso no necesariamente les dio ventaja porque el mercado, sus clientes, no estaban listos para entrar en el mundo digital.

Si bien se ha escrito mucho más sobre el proceso de transformación de la industria periodística y mediática, la industria de la comunicación comercial (agencias de publicidad, de relaciones públicas, de comunicación estratégica y agencias digitales) no escapa a este fenómeno. Autores como Lanas (2011), Martínez y Aguado (2014) y García Avilés (2009) han escrito sobre la cambiante realidad del sector y el impacto de las "nuevas y potentes formas de comunicación digital" (Lanas, 2011, p. 98) y también han señalado "la necesidad de reinventar la comunicación comercial" (Lanas, 2011, p. 98).

Lanas (2011) apunta que hay reordenación de las estructuras, nuevos organigramas y nuevos perfiles profesionales y agrega que:

En su evolución hacia nuevos modelos, las agencias de servicios plenos deben convivir con nuevas iniciativas empresariales que están irrumpiendo en el mercado con una vocación bien distinta, la especialización en áreas digitales concretas, dibujando finalmente un panorama hasta ahora inédito en la industria publicitaria (p.98).

En la era de la comunicación, cada vez en mayor medida, organizaciones grandes, medianas y pequeñas de todos los tipos requieren de estrategias, planes y productos de comunicación como herramientas para lograr competir mejor en el mercado. Para satisfacer esta creciente demanda se ha conformado una industria de la comunicación que, como se verá, es diversa en tipos de empresas y variedad de servicios.

A pesar de la importancia de la industria y de su trascendencia -el quehacer en comunicación no es hoy lo que fue hace tan solo unos años - no se ha hecho en Costa Rica una investigación que estudie cómo está constituida la industria de la comunicación y áreas afines en el país y que permita contestar preguntas como: ¿cuántas empresas ofrecen servicios de comunicación, relaciones públicas, publicidad, comunicación integrada, comunicación estratégica y comunicación digital? ¿qué tipo de empresas son? ¿qué servicios ofrecen? ¿quiénes son sus clientes? Interesa también entender cómo han afectado y cómo se viven los cambios que ha traído el vertiginoso desarrollo de las tecnologías de la información y la comunicación.

Esta radiografía pretende hacer un análisis general del quehacer comunicativo en Costa Rica y ser una forma de acercarse a un mejor entendimiento del futuro de esta área y la profesión.

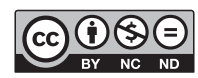


En el país, uno de los primeros esfuerzos para mapear el quehacer en el campo de la comunicación es el de Sandoval y Al Ghassani (1990) quienes construyeron un inventario de medios de comunicación que incluía medios impresos, radioemisoras, televisoras, agencias de noticias, salas de cine, agencias de publicidad, productoras audiovisuales y empresas de comunicación. Después de más de 25 años las comparaciones no resultan pertinentes; sin embargo, sí permiten evidenciar el desarrollo de la industria. La presente investigación no toma en cuenta a los medios de comunicación, únicamente a las empresas que ofrecen servicios de comunicación, relaciones públicas, publicidad y comunicación digital.

También es importante enmarcar esta industria como una opción creciente para desarrollar nuevos emprendimientos, específicamente en los últimos 10 años. Desde esta perspectiva Lebendiker, Herrera y Velázquez (2014) en su Reporte Global sobre el emprendimiento en Costa Rica mencionan que este país desarrolla emprendimientos en una economía basada en la eficiencia, donde los productos y servicios que se generan tienen un mayor grado de elaboración y son proclives a la presencia de alianzas estratégicas que permiten la asimilación de nuevas tecnologías y es aquí donde la industria de la comunicación ha sacado provecho.

De igual forma, y según este mismo estudio, en el 2012, cerca del 29\% de emprendedores establecidos clasificaban su emprendimiento en el sector de transformación, y un notable $61,4 \%$ en el sector orientado al consumidor. Es aquí donde se ubica el sector de la industria de la comunicación.

\section{Estudio}

El presente trabajo es el resultado de un proyecto de investigación inscrito en el Centro de Investigación en Comunicación (CICOM) de la Universidad de Costa Rica. El estudio hace un inventario de las empresas del campo de la comunicación y afines para hacer un diagnóstico de la industria en el país. Se incluyeron, por tanto, además de agencias de publicidad y agencias de relaciones públicas, agencias digitales y agencias de comunicación estratégica.

Para hacer un mapeo de la industria había que comenzar por identificar cuáles y cuántas empresas la constituyen. Esto de por sí resultó un reto porque no existe en el país un listado o una base de datos que pueda considerarse completa.

Al iniciar el estudio, en marzo 2015, el Colegio de Periodistas y Profesionales de la Comunicación de Costa Rica (COLPER) facilitó una base de datos con 28 agencias. El listado incluía a la mayoría de las más grandes, reconocidas y tradicionales agencias de publicidad y relaciones públicas del país. Por otra parte, un grupo de agencias de comunicación está agremiado a la Comunidad de Empresas de Comunicación de Costa Rica, una organización que reúne a algunas de las más grandes agencias y empresas de comunicación del país. De los 40 miembros que en noviembre de 2016 formaban parte de Comunidad (Carmen Mayela Fallas, comunicación personal, 15 de noviembre de 2016), 15 estaban incluidos en la lista del COLPER.

Entonces, además de los listados del COLPER y de Comunidad, se recurrió a diversas estrategias para lograr construir una base de datos de 136 agencias (ver Tabla 1). Algunas de las fuentes utilizadas fueron: información de graduados, graduadas y empleadores de la Escuela de Ciencias de la Comunicación Colectiva (ECCC) de la Universidad de Costa Rica, revistas especializadas, Facebook y validación con expertos. Además, resultó especialmente valioso incluir una pregunta al final del cuestionario para que los mismos representantes de las empresas de la industria refirieran a otras empresas menos conocidas.

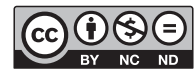


El grupo de empresas participantes se obtuvo a partir de un muestreo no probabilístico, ya que se realizó un muestreo por conveniencia que incluyó la colaboración voluntaria de 100 empresas del sector de la comunicación con sede en Costa Rica.

Durante los meses de abril a noviembre de 2016 se contactó por teléfono con cada empresa del sector y se verificó que su operación cumpliera los requisitos del perfil de empresas que serían tomadas en cuenta: organizaciones con constitución jurídica que emplearan a por lo menos una o un profesional en comunicación (periodista, publicista, relacionista público, productor audiovisual, comunicador).

Se concertó una cita telefónica con las personas representantes que ocupan los más altos cargos en sus empresas y conocen, por tanto, muy bien a su organización a la vez que cuentan con una visión privilegiada del negocio. En la mayoría de los casos los datos fueron proporcionados por representantes de las empresas en posiciones de liderazgo: propietarios, socios fundadores, socios, directores, presidentes y gerentes generales. La investigadora principal del estudio fue la encargada de hacer las preguntas a cada una de las personas representantes que contestó el cuestionario.

La información proporcionada por las empresas participantes se utilizó únicamente de manera agregada a fin de garantizar la confidencialidad de las organizaciones mapeadas (Tabla 1).

Tabla 1

Costa Rica: Mapeo de empresas de comunicación 2015-2016

Total de empresas identificadas

Empresas no contactadas o que no atendieron a la investigadora para contestar el cuestionario

Fuente: Elaboración propia con base en resultados de consulta a empresas de comunicación abril-noviembre 2016.

La principal limitación del estudio radica en que no se trata de una muestra aleatoria que sea representativa de la población (todas las empresas de la industria de la comunicación en Costa Rica). Es importante recordar que el muestreo es por conveniencia, así los resultados reflejan las características del grupo de empresas que contestó el cuestionario y no pueden generalizarse.

Por tratarse de un estudio exploratorio, se espera que los hallazgos estimulen la discusión en la industria y en la comunidad académica y que esto resulte en la generación de futuras investigaciones de mayor profundidad.

El trabajo se estructura en tres apartados: en el primero se realiza una caracterización general de las empresas de la industria. En el segundo apartado se documentan los aportes de la industria a la economía nacional analizando temas como empleo, encadenamientos, trabajo pro-bono y facturación. El tercer apartado explora el tema de lo digital: ¿desde cuándo ofrecen servicios digitales? ¿Cómo es esa oferta digital y cuál es la visión que tienen de lo digital hacia futuro?

\section{Caracterización de las empresas de comunicación}

\section{La evolución de la industria}

La primera empresa de la industria de la comunicación en el país, la agencia de publicidad Alberto H. Garnier, fue fundada en el año 1921. En todo el siglo XX, nacieron en el país 25

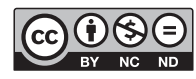


empresas de comunicación. Durante los primeros sesenta años, entre 1921 y 1981, se fundaron solamente siete agencias de publicidad. Ya en la década de los años 80 empiezan a nacer las primeras agencias de relaciones públicas. Durante esos diez años, de 1980 a 1989, la cantidad de empresas dedicadas a ofrecer servicios de comunicación se duplica, puesto que nacen otras siete agencias (Figura 1).

Durante la década de los años 90, que inició con 14 empresas de comunicación en el país, se sumaron 11 empresas más a la industria. Esto podría estar relacionado, en alguna medida, con que para los años 1993 y 1994 se dio un crecimiento importante en la cantidad de egresados del área publicitaria lo que eventualmente saturó dicho campo profesional, y esto pudo favorecer iniciativas profesionales independientes que se tradujeron posteriormente en empresas del área al finalizar la década (Sandoval, 1996, p.13-14).

No obstante, el panorama empieza a cambiar dramáticamente con el advenimiento del nuevo siglo, cuando ya ha iniciado el desarrollo de las tecnologías de la información y la comunicación. Entre los años 2000 y 2009 se crearon 35 agencias y entre los años 2010 y 2016 fueron fundadas 42 agencias más.

Esto quiere decir que más del $75 \%$ de las empresas encuestadas fue fundada a partir del año 2000. La Figura 1 ilustra claramente esta tendencia.

Figura 1

Costa Rica: Año de creación de las empresas de comunicación

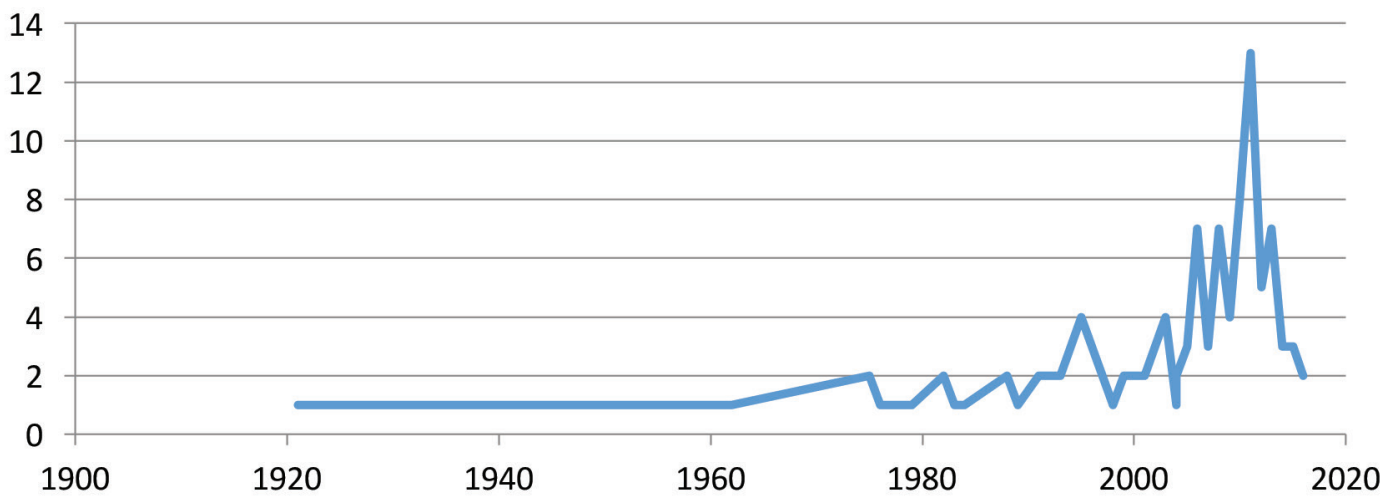

Fuente: Elaboración propia con base en resultados de consulta a empresas de comunicación abril-noviembre 2016.

Se le preguntó también a las personas representantes de las empresas si el capital de la empresa era costarricense, extranjero o una mezcla de ambos. Como se puede observar en la Figura 2, el 86\% de las empresas es de capital costarricense en su totalidad; tan solo $8 \%$ es de capital extranjero (la mayoría sucursales de empresas extranjeras en el país) y un $6 \%$ de capital mixto.

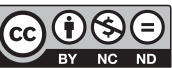


Costa Rica: Origen del capital de las empresas de comunicación

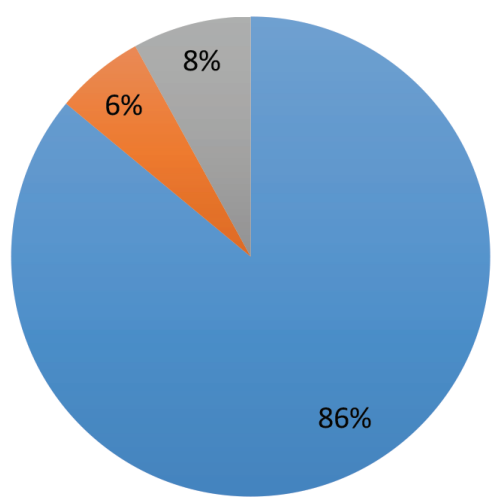

$100 \%$ costarricense

costarricense y extranjero

- $100 \%$ extranjero

Fuente: Elaboración propia con base en resultados de consulta a empresas de comunicación abril-noviembre 2016.

\section{El tamaño de las empresas de la industria}

La diversidad del tamaño de las empresas de la industria es muy grande. La muestra incluía desde microempresas de dos colaboradores hasta empresas grandes de más de 100 colaboradores (el valor máximo fue de 160 empleados). El 80\% de las organizaciones son micro y pequeñas empresas. El $16 \%$ son medianas y tan solo un $4 \%$ es empresa grande con 101 o más colaboradores. De las empresas medianas, un 75\% están en el rango inferior (entre 36 y 55 empleados). (Figura 3). La definición de micro, pequeña, mediana y gran empresa fue tomada del MEIC (Ministerio de Economía, Industria y Comercio) durante el mismo año del estudio (http//www.meic.go.cr).

En total, las empresas del sector contratan, en Costa Rica, a 2473 empleados.

Figura 3

Costa Rica: Tamaño de las empresas de comunicación (2016)

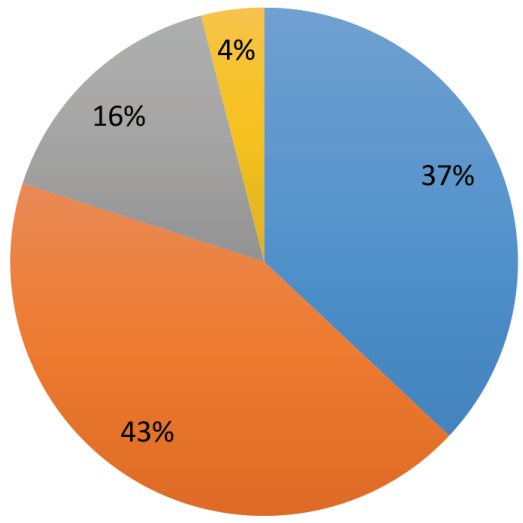

Micro (1-10 colaboradores)

Pequeña (11-35 colaboradores)

Mediana (36-100 colaboradores)

Grande (101 o más colaboradores)

Fuente: Elaboración propia con base en resultados de consulta a empresas de comunicación abril-noviembre 2016.

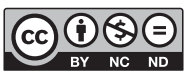

Rev. Reflexiones 98 (1) Enero-Junio, 2019: 39-60, E-ISSN: 1659-2859 


\section{Alcance geográfico de los servicios que ofrecen}

Los productos y servicios que crean y ofrecen las empresas de comunicación con sede en Costa Rica no circulan y se venden solamente en el país, sino que tienen un casi generalizado alcance regional y un fuerte alcance mundial. Tal y como se puede observar en la Figura 4, todas las empresas de la industria producen para Costa Rica pero, además, en casi un $80 \%$ de los casos también han vendido, venden o han circulado sus productos en la región centroamericana y del Caribe.

El $43 \%$ de las empresas mencionó a países latinoamericanos como México u otros países en Suramérica y el $31 \%$ ha trabajado proyectos para los Estados Unidos. Europa (8\%) y Asia (1\%) también fueron mencionados. En la categoría Otros (con 6\% de las menciones) se incluyen países como Canadá (tres menciones), Australia (una mención) y Nueva Zelanda (una mención), además del continente africano (una mención).

Figura 4

Costa Rica: Alcance geográfico de los servicios de las empresas de comunicación (2016)

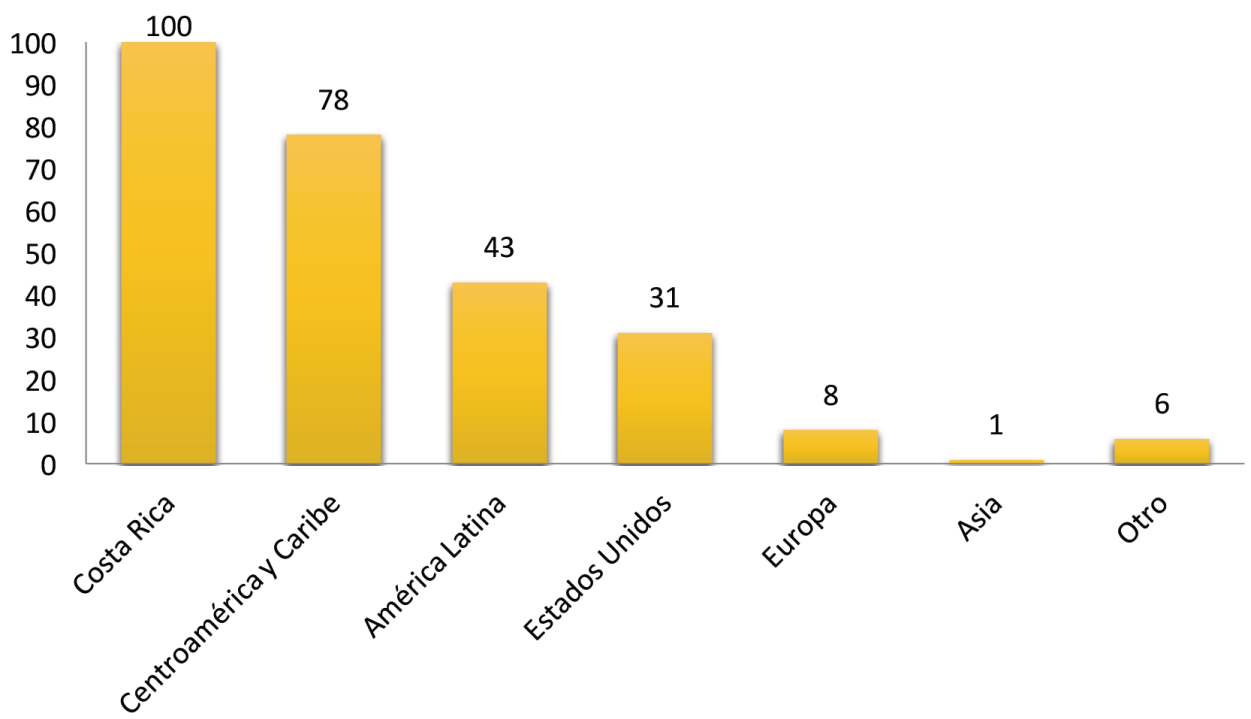

Fuente: Elaboración propia con base en resultados de consulta a empresas de comunicación abril-noviembre 2016.

\section{La organización y la oferta de servicios}

La convergencia de medios es un proceso facilitado por la implantación generalizada de las tecnologías digitales de la telecomunicación (García, 2009, p.102). En la industria periodística la convergencia ha traído cambios en la estructura organizativa de las empresas. No pocas salas de redacción han migrado a sistemas integrados. El presente estudio preguntó sobre el tema a los representantes de las empresas de la industria de la comunicación para conocer si se había dado un fenómeno similar. Este no fue el caso.

Mayoritariamente, las empresas de la industria siguen organizadas de la manera tradicional, por grupos de trabajo especializado o departamentos (cuentas, medios, digital, creatividad, etc.). La Figura 5 muestra que tan solo un $17 \%$ de las empresas está organizada en grupos de trabajo interdisciplinarios. El 12\% afirma ser demasiado pequeña y no tener división (todos hacen de todo).

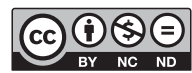


Estos datos son relevantes pues tal y como se evidenció en el Congreso sobre Publicidad Adweek 2017 (Fofstetter, Keith, Reardon, \& Sabol, 2017) al menos en el caso de las agencias de publicidad y relaciones públicas, no tendrán más alternativa que desarrollar equipos multidisciplinarios si desean tener una mayor permanencia en el mercado. Sus clientes, las marcas, cada vez están requiriendo de una mayor diversidad de disciplinas en el mismo equipo que les permita migrar hacia nuevas áreas (especialmente relacionadas con la tecnología) donde puedan establecer nuevos puntos de contacto y vínculo con los consumidores sin crear un entorno disruptivo alrededor de la publicidad de sus marcas.

Para Fofstetter (2017) la agencia debe ser capaz de ayudar a las marcas a conectarse con sus consumidores y debe estar conformada por un equipo de diferentes perfiles.

A pesar de lo que indican los datos, en algunas empresas - pocas- sí se han dado transformaciones; por ejemplo, uno de los directores afirmó:

Hemos hecho cambios: todos debemos saber de todo. Sí hay grupos, pero no por procesos. La división es más bien transversal. El cliente está al centro y los especialistas de negocio lo apoyan. Queremos alejarnos de lo lineal, de los silos. Ya ni siquiera hay paredes en la empresa. La idea es llegar a un punto en que todos sepamos de digital.

Otra de las personas representantes explicó que están organizados de la siguiente manera:

en duplas de trabajo que atienden a cuatro o cinco cuentas y hemos procurado ir especializando a cada dupla en cada una de las etapas. Para hacer esto se requiere de gente que esté dispuesta a aprender de manera continua.

Figura 5

Costa Rica: Modelo de organización de empresas de comunicación (2016)

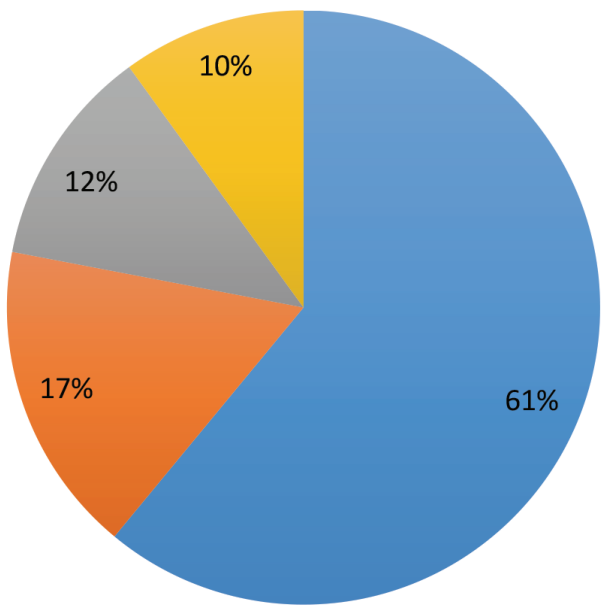

\author{
- Por grupos de trabajo \\ especializadoso \\ departamentos \\ - Por grupos interdisciplinarios \\ que atienden a cada cliente
}

- Somos demasiado pequeños y no tenemos división, todos hacemos de todo

Otro

Fuente: Elaboración propia con base en resultados de consulta a empresas de comunicación abril-noviembre 2016.

Lo que sí ha cambiado en gran medida es la oferta de servicios. Si bien entre las 100 empresas participantes había algunas especializadas, el estudio permite dibujar un panorama de una industria dinámica donde la línea divisoria entre las áreas de servicios como publicidad, relaciones públicas,

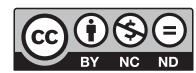


digital y BTL ("Below The Line”, es decir, que no emplea comunicación masiva) es cada vez más difusa porque las agencias reconocen la necesidad de brindar soluciones integrales a sus clientes.

Así, al preguntarle a las agencias por los servicios que ofrecen, se evidencia que empresas que nacieron como agencias de publicidad ahora también brindan servicios de relaciones públicas y, por supuesto, servicios digitales; de la misma manera que empresas de relaciones públicas también incursionan en BTL, en digital y, si el cliente lo requiere, incluso en publicidad.

Se confirma lo que plantea Weed (2017) en el sentido de que las agencias deben buscar integrar su trabajo con otras áreas, incluso con sus proveedores y hasta con otras agencias. Se hace necesario que los colaboradores deban salir a la calle, vivir la experiencia con el consumidor y ofrecer una solución completa a sus clientes que incluya muchas visiones y se traduzca en una experiencia más orgánica para el consumidor o la consumidora.

En el sector de agencias digitales es donde se evidencia más especialización, aunque, tal y como lo ilustra la explicación del socio fundador de una reconocida agencia digital, no son inmunes a la expansión:

Nacimos como agencia digital y por necesidades de los clientes nos fuimos expandiendo y nos convertimos en agencia tradicional. Las agencias tradicionales no se han desarrollado tanto en lo digital. Hoy es muy común que los clientes tengan dos o tres agencias. Antes tenían una sola pero hoy están buscando más la especialización. Nosotros compartimos clientes con muchas agencias grandes pero nosotros desarrollamos lo digital.

El socio fundador de otra reconocida agencia digital hizo hincapié en la especialización pero también apuntó hacia nuevas tendencias en el futuro:

En la gran mayoría de los casos todavía el mercado de lo digital funciona de manera compartida con lo tradicional: los grandes clientes tienen una agencia tradicional y una digital que les maneja esa parte. Esto está cambiando pero muy despacio. Nosotros tenemos algunos clientes, pero muy pocos, para quienes nosotros somos la única agencia, es decir, que tienen todo el presupuesto concentrado en digital.

Con respecto a este tema, tal y como se menciona en la ponencia "Marcas: los nuevos íconos de la cultura” (Eiserman, Bond, Fraga, Daryan, Shreffler, 2017), los clientes, es decir las marcas, están preocupadas por su futuro y le están exigiendo a los publicistas y sus agencias encontrar plataformas menos tradicionales donde la publicidad fluya de una forma más natural. Para Weed (2017) lo digital representa por mucho, la mejor plataforma para generar este acercamiento entre el consumidor y la marca.

Es interesante notar que la ampliación de la cartera de servicios no ha significado, necesariamente, que se haya multiplicado el tamaño de las organizaciones. Más bien, se han fortalecido los encadenamientos. Tanto en Costa Rica como en otras partes del mundo los encadenamientos son una forma inteligente de trabajar pues implican desarrollos con equipos multidisciplinarios que generan procesos más efectivos y con visiones más holísticas, lo que termina, por lo general, beneficiando a las marcas (Wacksman, Law y Radia, 2017).

En efecto, tal y como se verá más adelante en este artículo, el 90\% de las empresas afirmó que subcontrata algunos de los servicios que ofrece a sus clientes.

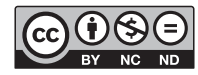

Rev. Reflexiones 98 (1) Enero-Junio, 2019: 39-60, E-ISSN: 1659-2859 


\section{El recurso humano}

Las agencias de publicidad, de relaciones públicas, de mercadeo y agencias digitales en el país contratan a personal con perfiles profesionales diversos.

Se les preguntó a los representantes de las empresas qué tipo de profesionales contrata la organización. Las tres categorías profesionales con más menciones fueron, en primer lugar, los diseñadores (mencionados por $86 \%$ de las empresas); en segundo lugar, los publicistas (72\% de las empresas los mencionan) y en tercer lugar, los administradores de empresas (mencionados por $62 \%$ de empresas).

Les siguen otros comunicadores, como periodistas y relacionistas públicos (mencionados por $58 \%$ de las empresas), los mercadólogos (49\%) y profesionales en computación, programadores e informáticos (48\%). Las personas de producción audiovisual solamente fueron mencionadas por el $38 \%$ de las empresas; esto puede deberse a que, como se verá más adelante, la producción audiovisual es uno de los servicios que las empresas de la industria más contratan a proveedores externos.

Se le preguntó, también, a las personas representantes de las empresas de comunicación por el peso relativo de algunos factores a la hora de hacer una nueva contratación. La mayoría (32 menciones) aseguró que lo más importante es la experiencia profesional en el campo. Le siguió en el primer lugar de importancia el portafolio y luego las referencias o recomendaciones.

En un distante segundo nivel, con la mitad de las menciones o menos, se encuentran los factores relacionados con la formación universitaria: solamente 33 representantes de las empresas indicaron que tener un título universitario era un factor que ocupara el primer, segundo o tercer lugar de importancia a la hora de hacer una nueva contratación y solamente 17 personas afirmaron que para la empresa es importante la universidad en la que estudia o de la que se graduó una persona que pretenden contratar. La categoría "Otros factores" estuvo por encima de la universidad. En "Otros factores" sobresalió, ante todo, la actitud: tener buenas relaciones interpersonales, saber trabajar en equipo, ser constructivo y respetuoso y contribuir a un buen ambiente en la empresa, entre otros. Algunas personas también mencionaron idioma y habilidades digitales. Es interesante mencionar que en el Congreso sobre publicidad, realizado en New York, la ponencia titulada Self-Disrupt: Creating the next agency model (Wacksman, Law y Radia, 2017) no menciona ni la experiencia profesional ni el portafolio como aspectos tan trascendentales sino la capacidad de crear relaciones de colaboración entre pares, así como con los clientes y marcas. Asimismo plantea los seis aspectos esenciales que un buen equipo en la agencia debe manejar: el propósito de la marca, la innovación, el ecosistema de trabajo, la experiencia del consumidor, la comunidad y la cultura. Se cree que el nuevo modelo en las agencias debe contemplar equipos que trabajen sobre estas bases para ayudar posteriormente a desarrollar junto con sus clientes innovaciones a través de start-ups o nuevos emprendimientos con un alto componente tecnológico.

Wacksman (2017) sostiene que el principal problema en los equipos de trabajo es la ejecución de los procesos por falta de esas habilidades blandas, que no logran conectar con el entorno comunicacional y conectar con el diseño de las marcas. En síntesis, las llamadas habilidades blandas son más importantes que las duras tanto en este como en otros mercados como el estadounidense. En lo que sí parecen coincidir es en la relativa poca importancia que se le da a un título universitario como tal.

\section{Encadenamientos productivos}

La industria de la comunicación contribuye a la economía nacional a través de los encadenamientos productivos, una práctica que, según la investigación, tiene fuerte presencia en la industria.

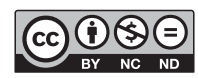


Solamente 10 empresas aseguraron que todos los servicios que ofrecen los realizan con personal en planilla. Es decir, el 90\% de las empresas de la industria subcontratan a proveedores externos para cumplir satisfactoriamente con la gama de servicios que ofrecen.

En palabras de uno de los representantes de la industria consultados: "Contratar a proveedores externos nos da capacidades que no tenemos en la agencia, escogemos los mejores del mercado para ofrecer el mejor servicio, esto al menor costo para la empresa y para nuestros clientes".

La Tabla 2 detalla las principales razones que llevan a las empresas de la industria a contratar a proveedores externos.

Tabla 2

Costa Rica: Razones de las empresas de comunicación para contratar a proveedores externos (2016)

Permite resolverle a los clientes todas las necesidades relacionadas con comunicación.

Son servicios especializados con poco volumen.

Cuando son servicios que no son el negocio principal o el fuerte de la empresa o áreas de conocimiento que no se han desarrollado en la empresa.

- Una diversidad de proveedores de confianza que trabaja con calidad da la opción de seleccionar de acuerdo a lo que el cliente necesita (calidad, precio, estilo).

Permite mejor control de costos, más eficiencia y mayor competitividad en cuanto a tarifas.

Los tiempos de respuesta son mejores.

Cuando hay muchos proyectos o proyectos grandes y no dan abasto los recursos de la empresa.

Inversión en equipamiento costosa.

Fuente: Elaboración propia con base en resultados de consulta a empresas de comunicación abril-noviembre 2016.

Los servicios subcontratados que se mencionan con mayor frecuencia por los representantes de la industria fueron: producción audiovisual, programación, eventos, fotografía e impresión, entre otros.

Varios representantes de empresas explican que le han apostado a la subcontratación como modelo de negocio. Una persona representante de una pequeña agencia-estudio afirmó:

Creemos mucho en el networking; creemos que las estructuras grandes y pesadas no son el futuro. No consideramos que el modelo de agencia gigante con muchos departamentos sea el más ágil ni el del futuro. Creemos en el estudio pequeño que puede ofrecer mucho talento y que trabaja a través de una red de colaboradores.

Otro representante, haciendo eco de la manera cómo funcionan varias de las agencias participantes en las que se subcontrata la mayoría de los servicios, explicó: "Nosotros pretendemos ser los directores de orquesta y contratamos a los instrumentos que necesitamos". Una tercera persona, representante de una agencia que está interesada en incursionar en este modelo de negocio (subcontratación), señaló: "Queremos ser más pequeños y subcontratar más. Queremos solamente vender pensamiento. El objetivo es encadenar". Se trata, sin lugar a dudas, de una tendencia que debe investigarse en mayor profundidad.

Tal y como menciona Fofstetter (2017), los procesos de colaboración en las agencias son cada vez más valiosos tanto entre los mismos integrantes como con otros proveedores o bien otras agencias. Por ende, la subcontratación no es ajena a esta misma industria en otras partes del mundo. Para Sean Reardon (2017) lo más importante sigue siendo ayudarle al cliente a estar más cerca de su consumidor independientemente del modelo de contratación que se tenga en cada agencia.

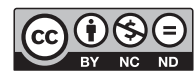

Rev. Reflexiones 98 (1) Enero-Junio, 2019: 39-60, E-ISSN: 1659-2859 
Además de la satisfacción integral de las necesidades de los clientes, los encadenamientos permiten la multiplicación de la industria, no obstante, también tienen implicaciones en cuanto a seguro social y posibilidades de garantizar derechos laborales que no fueron objeto de estudio de la presente investigación, pero que deben abordarse en otros trabajos en el futuro.

\section{La clientela}

La industria de la comunicación está constituida no sólo por la oferta sino también por la demanda de los servicios. Para conocer sobre ese otro aspecto del negocio, se le preguntó a las personas representantes de las empresas sobre su clientela.

La Figura 6 muestra sus respuestas cuando se les solicitó que describieran, aproximadamente, cómo estaba compuesta su cartera de clientes. Las empresas nacionales grandes y las corporaciones transnacionales o empresas internacionales, fueron mencionadas en mayor medida: 68 empresas indicaron que tienen entre su cartera de clientes a empresas nacionales grandes y 60 a corporaciones transnacionales o empresas internacionales. Les siguieron en cantidad de menciones las empresas nacionales medianas (57 menciones) y las empresas nacionales pequeñas (54 menciones). Menos de una tercera parte (29 menciones) de las empresas de la industria trabaja para instituciones del sector público y tan solo 14 empresas cuentan con ONGs en su cartera de clientes.

Para responder a la pregunta, cada una de las empresas debía dividir el 100\% de su cartera de clientes entre los porcentajes aproximados que cada sector representaba para su cartera. El promedio más alto fue para las corporaciones transnacionales o empresas internacionales (47.55). En promedio, las empresas nacionales (pequeñas, medianas y grandes) representan, cada una, una tercera parte de la cartera de clientes de las empresas de la industria: 32.93 las pequeñas, 32.65 empresas medianas y 35.37 empresas grandes.

Figura 6

Costa Rica: Mezcla de la composición de la cartera de clientes de las empresas de comunicación (2016)

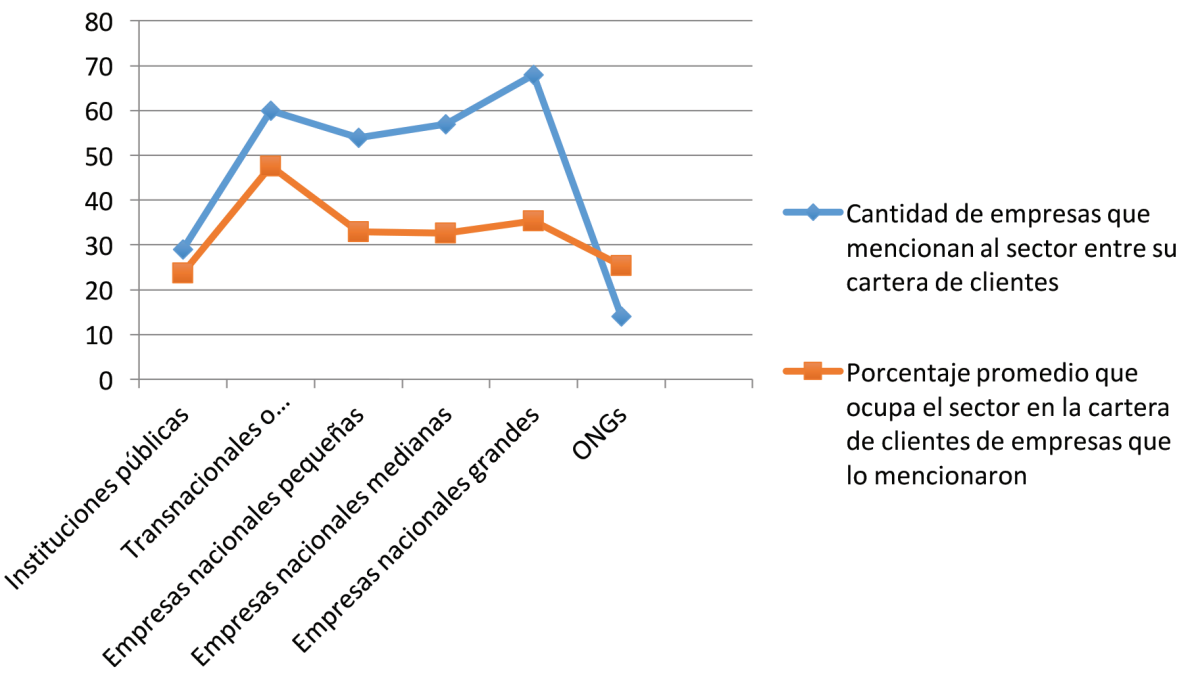

Fuente: Elaboración propia con base en resultados de consulta a empresas de comunicación abril-noviembre 2016.

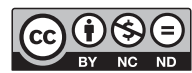

Rev. Reflexiones 98 (1) Enero-Junio, 2019: 39-60, E-ISSN: 1659-2859 
También se consultó a cuántos clientes habían atendido en el año 2015. La mayoría de las empresas de la industria (59\%) atendió entre 11 y 30 clientes. El $26 \%$ atendió a 31 o más clientes y solamente un 15\% le dio servicios a diez o menos clientes durante el 2015 (ver detalle en Figura 7). Un cálculo conservador (utilizando promedios para todas las categorías y 55 clientes para la categoría más alta) arroja un estimado de 2350 clientes atendidos durante el año 2015 por las 100 empresas de la industria que participaron en el presente estudio.

Figura 7

Costa Rica: Cantidad de clientes de las empresas de comunicación (2015)

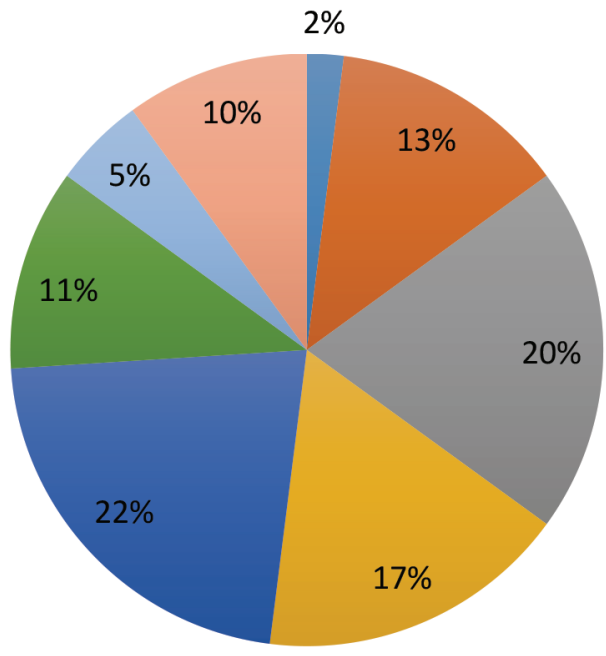

Menos de 5

De 5 a 10

De 11 a 15

De 16 a 20

De 21 a 30

De 31 a 40

De 41 a 50

Más de 51

Fuente: Elaboración propia con base en resultados de consulta a empresas de comunicación abril-noviembre 2016.

\section{La facturación}

Con el fin de dimensionar el peso de la industria de la comunicación en la economía nacional, se le preguntó a las empresas participantes por un aproximado de su facturación en el año 2015 (Figura 8).

El 20\% de las empresas facturó menos de US\$100 mil; se trata, en mayor medida, de empresas micro (16) y pequeñas (4). El 35\% de las empresas facturó entre US\$101 mil y US\$500 mil: 13 microempresas (1-10 colaboradores) y 2 empresas pequeñas (11-35 colaboradores) facturaron entre US\$101 mil y US\$250 mil, mientras que 4 microempresas, 15 empresas pequeñas y 1 empresa mediana (36-100 colaboradores) facturaron entre US\$251 mil y US\$500 mil.

Un 14\% de las empresas facturó entre US\$501 y US\$1 millón. En relación con la cantidad de colaboradores, estaban en este segmento 2 microempresas, 8 empresas pequeñas y 4 empresas medianas.

El 26\% facturó más de US\$1 millón. Como se puede desprender de la Tabla 3; es interesante notar que no se trata únicamente de las empresas con más colaboradores ya que este porcentaje también incluye empresas pequeñas con (11-35 colaboradores) y medianas (36-100 colaboradores): 13 empresas pequeñas, 11 empresas medianas y 2 empresas grandes aseguraron estar en esta categoría.

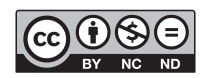


La Tabla 3 detalla más datos relevantes en relación con la facturación: las empresas que facturan más de US\$1 millón no son solamente las empresas con más años de trayectoria, pues 14 de las empresas que nacieron a partir del año 2006 (seis de ellas creadas a partir del año 2010) están en esta categoría.

De igual manera, hay empresas con relativamente pocos clientes que tienen alta facturación: cinco empresas con 15 o menos clientes están en el rango de facturación mayor al millón de dólares. No obstante, a pesar de estas notables excepciones, por lo general, entre menos clientela más baja la facturación y entre más clientela, más alta.

Un cálculo conservador del total de facturación de las 95 empresas que contestaron a la pregunta sobre sus ingresos en el año 2015 (utilizando los promedios de cada rango y apenas un estimado de US $\$ 1.5$ millones para las empresas que se colocaron en el rango superior de más de US\$1 millón) arroja que la industria aportó a la economía nacional aproximadamente US\$61.125.000.

Figura 8

Costa Rica: Facturación de las empresas de comunicación (2015)

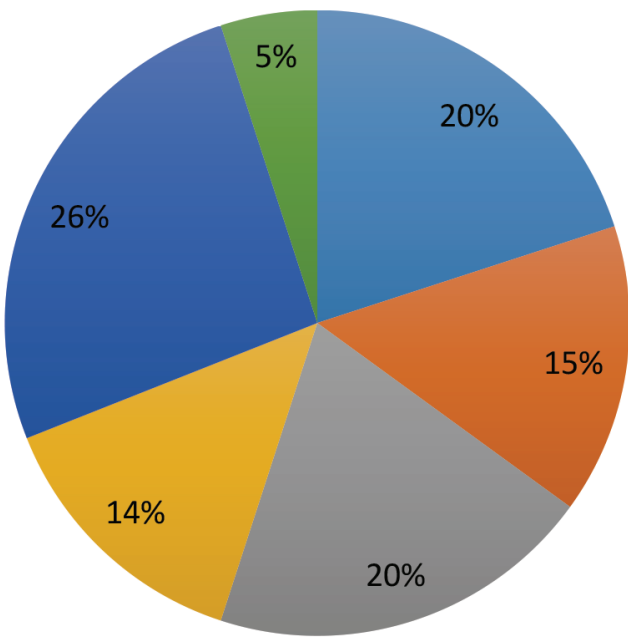

Menos de $\$ 100 \mathrm{mil}$

- Entre \$101 mil y \$250 mil

Entre \$251 mil y \$500 mil

— Entre \$501 mil y \$1 millón

- Más de \$1 millón

- Sin respuesta

Fuente: Elaboración propia con base en resultados de consulta a empresas de comunicación abril-noviembre 2016.

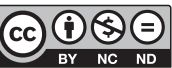


Tabla 3

Costa Rica: Facturación de las empresas de comunicación según cantidad de colaboradores y colaboradoras, año de fundación y cantidad de clientes por sector (2015)

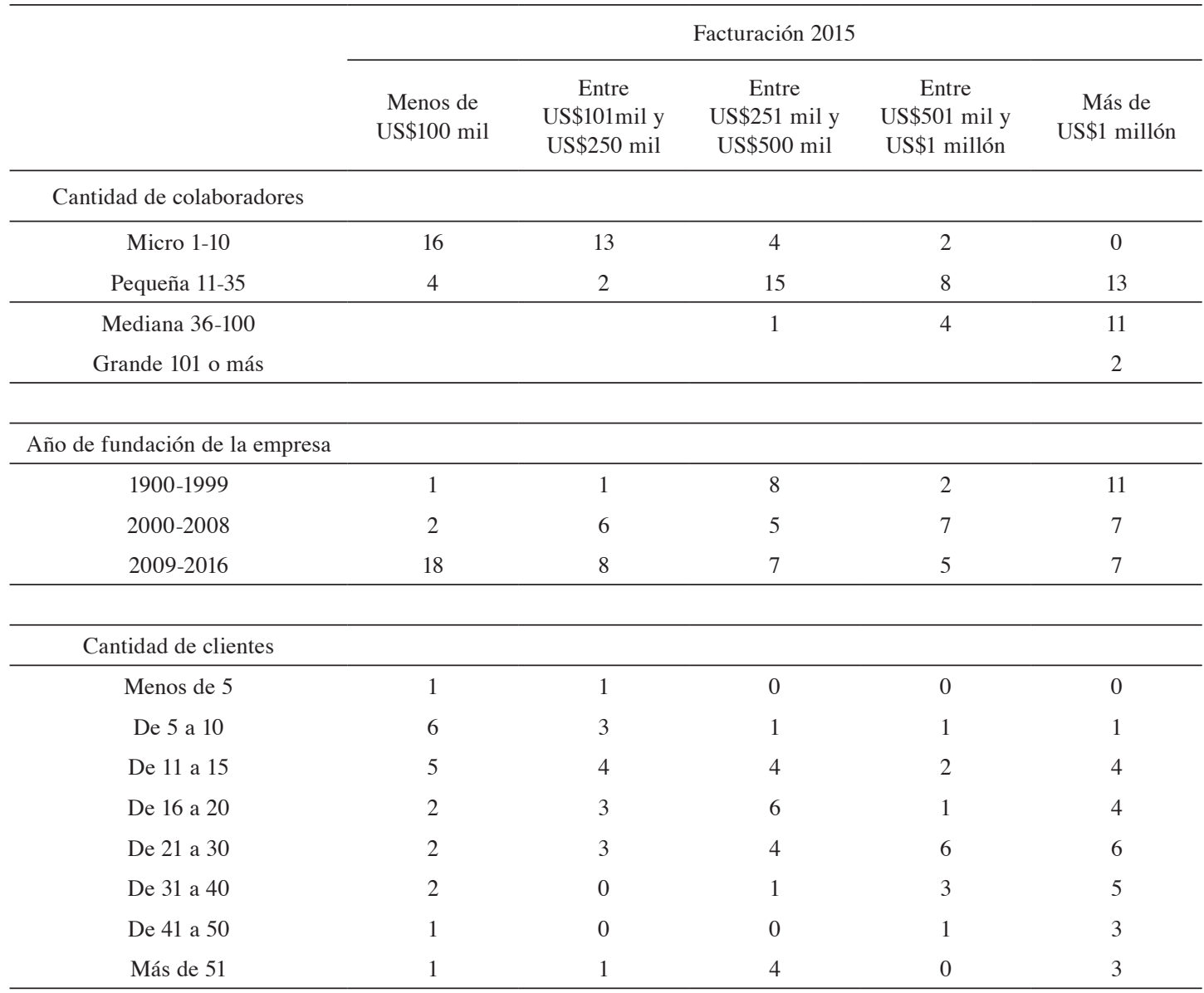

Fuente: Elaboración propia con base en resultados de consulta a empresas de comunicación abril-noviembre 2016.

\section{Trabajo pro-bono}

Las empresas de la industria de la comunicación también contribuyen con la economía nacional a través de la donación de su trabajo en proyectos para organizaciones sin fines de lucro o con muy pocos recursos, conocido como trabajo pro-bono.

Se trata de una práctica muy extendida en la industria: casi las tres cuartas partes de las empresas afirmaron que tienen objetivos de responsabilidad social que incluyen la atención de clientes pro-bono.

En el año 2015, las empresas que realizaron trabajo pro-bono apoyaron a 70 clientes. La práctica más usual (en el caso del $69 \%$ de las empresas) es tener uno o dos proyectos o clientes pro-bono como máximo. La mayoría de las empresas, el 43\%, colaboró con dos proyectos pro-bono en el año 2015, seguido de un $26 \%$ que apoyó a un proyecto durante ese año. El $12 \%$ de las empresas tuvo tres clientes

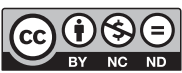


pro-bono, el $7 \%$ cuatro proyectos y el $4 \%$ cinco proyectos. Un $8 \%$ de las empresas que hacen trabajo pro-bono afirman haber colaborado con seis o más clientes en el año 2015.

$\mathrm{Al}$ preguntarle a las personas representantes de las empresas a cuánto podría haber ascendido el monto facturado de no haber sido trabajo pro-bono, la mayoría (27\%) se colocó en la categoría "entre US\$5 mil y US\$15 mil”. Un 19\% colaboró con montos que oscilan entre los US\$16 mil y US\$25 mil y un $17 \%$ con montos entre US $\$ 26$ mil y US\$50 mil. Si se saca un promedio de los aportes de este $63 \%$ de empresas (44 organizaciones), en total colaboraron con casi un millón de dólares (US\$ 912 mil) en trabajo pro-bono en el año 2015 (Figura 9). Algunas empresas (10 organizaciones que representan un $14 \%$ ) contribuyeron con US\$5 mil o menos, pero también un 14\% aportó trabajo pro-bono por montos entre US\$51 mil y US\$100 mil. Seis empresas (un 9\%) afirmaron haber contribuido con trabajo probono equivalente a más de US\$100 mil durante el año 2015.

Un cálculo conservador del total del aporte de las empresas de la industria en trabajo pro-bono durante el año 2015 permite estimar el aporte en más de US\$2,250 millones.

Figura 9

Costa Rica: Aporte de trabajo pro-bono de las empresas de comunicación (2015)

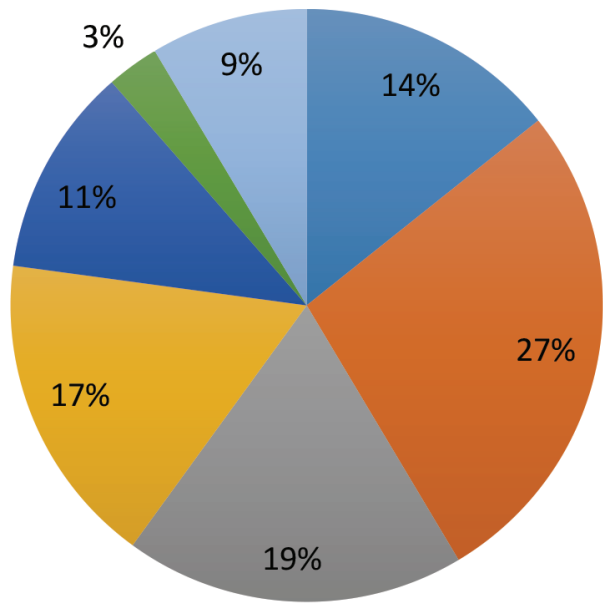

$\$ 5$ mil o menos

Entre $\$ 5$ mil y $\$ 15$ mil

Entre \$16 mil y \$25 mil

Entre \$26 mil y \$50 mil

Entre \$51 mil y \$75 mil

Entre \$76 mil y \$100 mil

Más de $\$ 100$ mil

Fuente: Elaboración propia con base en resultados de consulta a empresas de comunicación abril-noviembre 2016.

\section{La industria de la comunicación y lo digital}

Desde mediados de la década de los años noventa, prácticamente al mismo tiempo en que da inicio el desarrollo de Internet en el país, las primeras agencias empiezan a incursionar en el área digital (ver Figura 10). La primera agencia que asegura empezó a ofrecer servicios en el área digital lo hace en el año 1994. La primera agencia digital nace en el año 1995. Por supuesto que, esos primeros servicios eran muy distintos de lo que hoy se entiende por digital. Se trataba de los primeros sitios web. Una de las personas representantes explicó que eran algo así como "brochures electrónicos" pues se trataba de páginas web muy sencillas. Otro servicio que ofrecían las

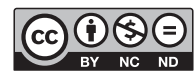


agencias en aquellos primeros años era el de correo electrónico (envío, recepción y respuestas a correos) porque la clientela no lo tenía.

La Figura 10 muestra el año en que cada una de las empresas del estudio empezó a ofrecer servicios en el área digital. La línea azul representa a todas las agencias y la línea roja representa únicamente a las agencias digitales (33 en total).

Figura 10

Costa Rica: Año de inicio de oferta de servicios digitales de las empresas de comunicación

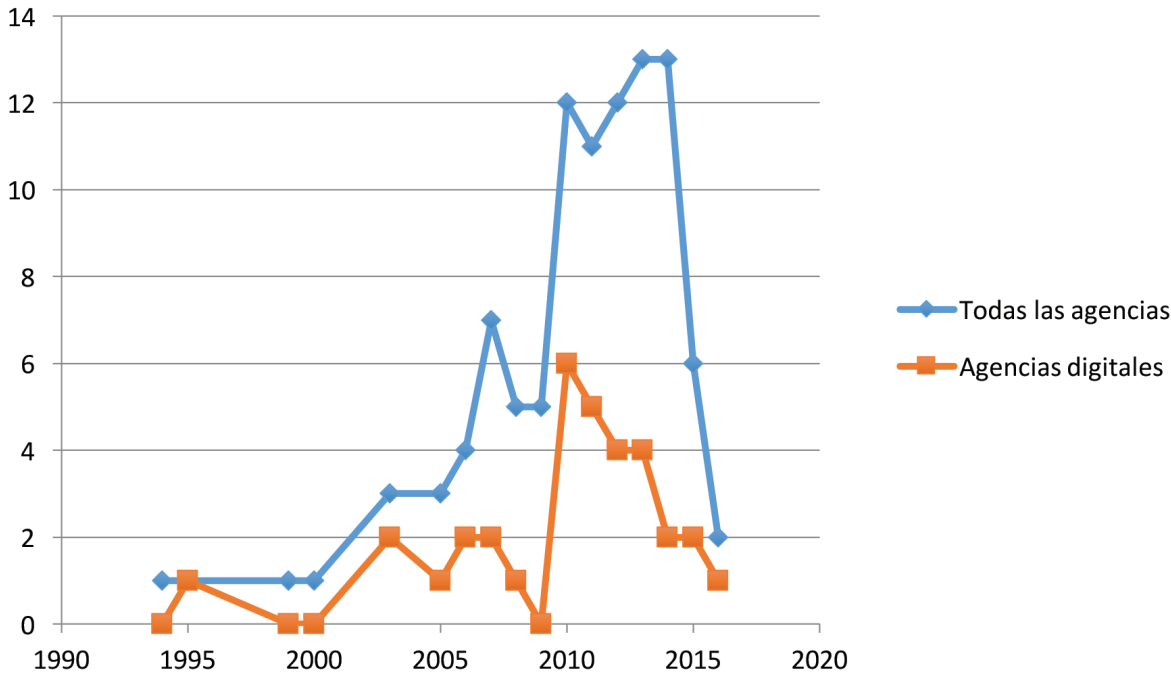

Fuente: Elaboración propia con base en resultados de consulta a empresas de comunicación abril-noviembre 2016.

Durante los primeros diez años (1994-2003), solamente siete agencias empezaron a ofrecer servicios digitales, incluyendo tres agencias que nacieron como agencias digitales. A partir del año 2005, empieza un crecimiento sostenido, pero todavía tímido (en el periodo 2005-2009, 24 agencias, incluyendo seis agencias digitales, sumaron a su oferta de servicios lo digital). A tono con lo que señalan Lanas (2011) y Martínez y Aguado (2014), el año 2010 marca el despegue pronunciado de la oferta de servicios digitales. En el periodo 2010-2014 fueron 61 las agencias que incorporan esta nueva área a los servicios que ya ofrecían, incluyendo las 21 agencias digitales que nacieron en el periodo.

De las 100 empresas de la comunicación que participaron en el estudio, 33 son organizaciones que nacieron como agencias digitales. Al resto de agencias (67), se les preguntó si consideraban que lo digital había tenido un impacto significativo en el quehacer de la empresa. El 30\% aseguró que "en gran medida" el impacto de lo digital había sido significativo, que habían tenido que reinventarse y había implicado reestructuración de la empresa. La mayoría, casi un 50\%, afirmó que "en alguna medida" el impacto de lo digital había sido significativo: se integró lo digital a la gama de servicios que ya se ofrecían y se hicieron algunos ajustes en la manera como trabajaba la empresa. Solamente un $16 \%$ aseguró que el impacto de lo digital no había sido "para nada" significativo, ya que no implicó grandes cambios en la empresa.

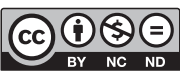


Las personas participantes en el estudio coinciden con los autores que han escrito sobre el tema (como Lanas, 2011; Martínez y Aguado, 2014 y García Avilés, 2009) en que lo digital es cada vez más importante en el mundo de la comunicación comercial.

Como bien lo menciona Weed (2017) la sociedad está migrando de grandes medios (televisiónradio-prensa), grandes cadenas minoristas y enormes presupuestos en publicidad y mercadeo a medios digitales, específicamente al teléfono móvil, con presupuestos mucho más accesibles y al e-commerce. Lo digital, según el autor, implica trasladar al consumidor al centro de la ecuación. Es decir, esto conlleva entender el cambio en la trayectoria de su proceso de consumo y en la forma en cómo toma decisiones este individuo, pues de lo contrario se perderá todo vínculo con este consumidor.

En Estados Unidos un tercio de las personas menciona que su principal forma de entretenimiento la lleva a cabo a través del móvil (Weed, 2017), esto eventualmente podría ir incrementando y transformándose en una tendencia. Por lo tanto, para las agencias de comunicación es indispensable desarrollar mejores formas de engagement (vincularse) con los consumidores y las consumidoras a través de este tipo de plataformas y esto, necesariamente, implica relacionarse cada vez más con lo digital.

Las personas representantes de las empresas consultadas señalan que ha habido un proceso de democratización (que no necesariamente ha favorecido al sector de las agencias formales, pero cuya presencia es innegable). Uno de los participantes lo resumió así:

Las posibilidades de producir antes eran solo para los clientes que tenían grandes presupuestos, ahora esas posibilidades de producción se han acercado para todos. Se eliminaron las barreras de ingreso a la industria. Antes había que invertir en infraestructura, en la red, en la central telefónica... ahora ya no, ahora solo se necesita un teléfono inteligente.

Otra persona dijo lo siguiente:

El mundo digital es maravilloso: no solo te obliga a reinventarte, a estudiar, a capacitarte, sino que también es absolutamente democrático para proveedores y para clientes: ahora tenemos clientes que antes no podían contratar a una agencia de publicidad; igual que hay muchachos que se pagan los estudios ofreciendo estos servicios.

Además de este fenómeno de ubicuidad de lo digital, destacan los comentarios relacionados con los crecientes presupuestos que la clientela de la industria de la comunicación invierte en digital. Según la mayoría de participantes en el estudio, se trata de una tendencia muy reciente, pues el inicio de lo digital en el país fue más bien lento. Varias personas mencionaron la edad de los gerentes de mercadeo de sus clientes como un factor importante que explica el lento despegue de lo digital en el país. Una de ellas lo explicó así:

Los tomadores de decisiones en las empresas son personas mayores de 40 años y eso hizo que el inicio fuera lento. Entre más jóvenes sean los tomadores de decisiones más abiertos están a experimentar y a entender el proceso digital. La industria se va a desarrollar de 'manéjeme las redes', que es lo que está privando en este momento, a estrategias más integrales. Lo que viene será más rápido que en el inicio.

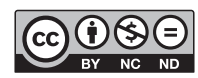

Rev. Reflexiones 98 (1) Enero-Junio, 2019: 39-60, E-ISSN: 1659-2859 
En efecto, hubo consenso entre quienes participaron en el estudio que la facturación cada vez se vuelca más a digital, “ya es y será cada vez con más fuerza, el corazón de las campañas”, apuntó un representante de la industria.

Las personas consultadas explicaron que se ha logrado que los presupuestos se vayan diversificando y con estos las prioridades. Así, si un presupuesto dedica un 5\% a la pauta digital, un gerente de marca probablemente no le da tanta importancia, pero si representa un $30 \%$, el gerente de marca le pone más atención a esa inversión.

Adicionalmente, para los representantes de la industria cada vez más los clientes comprueban la efectividad que asegura lo digital, sobre todo en relación con el rendimiento y el control de su pauta.

Por esa razón, no es de extrañar que uno de los representantes hiciera la siguiente predicción:

Todo se va a volver digital en los próximos 15 o 20 años. Antes pensábamos que iba a ser más rápido la evolución a lo digital, pero el mismo mercado ha ido lentamente. Pero ahora sí creen en redes sociales, optimizan páginas web... Hoy el 65\% del trabajo en la empresa es de medios tradicionales, en los próximos 5 años se va a invertir la proporción.

No obstante, para una de las personas que contestó el cuestionario: "en Costa Rica todavía lo digital está en pañales porque los clientes entienden por digital nada más Facebook. Las agencias tienen que educar a los clientes sobre todas las potencialidades de lo digital".

En este sentido, se aprecia una sustancial diferencia entre lo que se desarrolla en otras áreas del mundo, específicamente en Estados Unidos, donde para la industria de la comunicación es claro que lo digital es por mucho la prioridad tanto de las marcas como de las agencias.

La industria de medios tradicionales está tratando de reinventarse para poder competir con el mundo digital y de hecho si lo hace sería a través de éste, que es donde los consumidores y las consumidoras desean estar e invierten buena parte de su tiempo (Weed, 2017). El gran reto en este caso, es cómo lograr que la publicidad en redes sea más orgánica y fluya sin entorpecer los intereses de las personas consumidoras y no les genere una disrupción negativa ni en su contenido ni en su forma.

Se le preguntó a todas las personas que contestaron el cuestionario sobre su visión de lo digital hacia futuro. Sus respuestas permiten dirigir el foco hacia algunas tendencias importantes.

En primer lugar, fueron reiterados los llamados no solamente a cuidar el contenido, sino a destacar en su producción. Una persona explicó que el mercado y la clientela exigen cada vez más un mejor desarrollo de contenidos: "Ya no es tanto el community management porque los clientes lo tienen internamente, pero sí contratan a las agencias para la generación, creatividad y producción de contenido".

Relacionado con el contenido está el tema de las experiencias, pues no basta con un contenido tradicional. Uno de los participantes lo expresó así:

Hacia futuro debe desarrollarse en primer lugar, contenido interactivo; en segundo lugar, contenido integrado (en todos los medios). El objetivo es capturar la imaginación del consumidor. Las claves son "engagement" y experiencias.

No sorprende que otra de las tendencias que destacó entre las respuestas de los participantes fue la de big data o, como lo dijo uno de los representantes de la industria "la inteligencia de data". Otro de ellos explicó que en su agencia el cambio más importante había sido hacia el enfoque de datos:

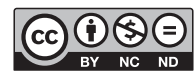

Rev. Reflexiones 98 (1) Enero-Junio, 2019: 39-60, E-ISSN: 1659-2859 
Pasamos de ser una agencia que ofrecía contenido de redes sociales y administración, a tener un enfoque importante en big data. El salto es hacia poder entender cómo los datos se pueden convertir en insights (ideas) y retorno de inversión para el cliente.

El enfoque en big data exigirá algo que se ha hecho presente cada vez con mayor fuerza en la industria: el trabajo en equipos interdisciplinarios, incluyendo profesionales en estadística y en informática y computación.

Otras áreas de desarrollo que fueron mencionadas por los representantes de la industria fueron: realidad virtual, Internet de las cosas, más énfasis en la interface y la experiencia de usuario, lo digital y el punto de venta, geolocalización y, por supuesto, desarrollo de aplicaciones (apps).

El sector digital presenta, sin duda alguna, grandes retos para la industria y, también, para la academia. Se debe tener el dinamismo con el que el mismo campo cuenta, mantenerse informado, estudiar, capacitarse, experimentar e innovar.

\section{Reflexiones finales}

Esta radiografía de las empresas de comunicación presenta un análisis general del quehacer comunicativo en nuestro país y pretende ser una forma de acercarse a un mejor entendimiento del futuro de esta área y de la profesión.

Esta investigación nos permitió retratar a muchas de las empresas de la industria de la comunicación. En primer lugar, se trata de un sector que se ha multiplicado, sobre todo, a partir del nuevo siglo y el desarrollo de Internet y la era digital: el $75 \%$ de las 100 empresas que participaron en el estudio nació en el siglo XXI. A pesar de que son empresas relativamente jóvenes, la mayoría brinda servicios regionales (Centroamérica y el Caribe). Casi la mitad (43\%) también trabaja o ha trabajado en países de América Latina y el alcance de una tercera parte (31\%) llega hasta Estados Unidos.

La industria es, casi en su totalidad, de capital exclusivamente costarricense (86\%) y hay una marcada presencia de empresas pequeñas: el $80 \%$ son micro y pequeñas empresas (con menos de 35 empleados). En total, las empresas del sector contratan, en Costa Rica, a 2473 empleados.

La diversidad del tamaño de las empresas de la industria es muy grande. La muestra incluía desde microempresas de dos colaboradores hasta empresas grandes de más de 100 colaboradores (el valor máximo fue de 160 empleados). El 80\% de las organizaciones son micro y pequeñas empresas. El $16 \%$ son medianas y tan solo un $4 \%$ es empresa grande con 101 o más colaboradores. De las empresas medianas, un $75 \%$ están en el rango inferior (entre 36 y 55 empleados).

En todas las empresas predomina el trabajo interdisciplinario con presencia, sobre todo, de diseñadores gráficos, periodistas, relacionistas públicos, publicistas, profesionales en computación, mercadólogos y administradores.

La industria de la comunicación en el país, aunque puede considerarse relativamente pequeña si se le compara con otras como la turística o la de la construcción, es un sector estratégico en la economía nacional. Una cuarta parte de las empresas que participaron en el estudio afirmaron haber facturado más de un millón de dólares (US) en el año 2015. En total, un cálculo conservador del aporte de todas las empresas del sector durante el año 2015 asciende a US\$ 61,125 millones. Ese mismo año, atendieron, por lo menos, a 2350 clientes de los más diversos sectores: empresas internacionales y transnacionales; empresas nacionales grandes, medianas y pequeñas; y, en menor medida sector público y ONGs.

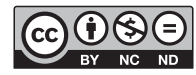


La industria también aporta a la economía nacional a través de encadenamientos, una práctica con fuerte presencia en el sector puesto que el $90 \%$ de las empresas de la industria subcontratan a proveedores externos para cumplir satisfactoriamente con la gama de servicios que ofrecen.

Según representantes de las empresas participantes en el estudio, el "outsourcing" permite satisfacer de manera integral las necesidades de los clientes, con calidad y de la manera más eficiente (desde el punto de vista de costos) para las empresas. Esto ha permitido que muchos proveedores de estos servicios sean parte del engranaje que compone la industria; sin embargo, también tiene implicaciones relacionadas con la seguridad social y la garantía de derechos laborales de cientos de personas que debe ser objeto de investigación en el futuro.

Otro aporte que se hace desde el sector es el de donaciones de trabajo (pro-bono): 70\% de las empresas afirma que tiene como práctica realizar trabajo pro-bono. En el año 2015, atendieron a 70 clientes pro-bono y aportaron aproximadamente US\$2,250 millones.

El campo digital es el de mayor crecimiento en la industria y el que presenta mayores retos por su dinamismo, variedad y demandas de actualización. La migración de las marcas al entorno digital parece inevitable, por lo que las agencias que asesoran a estas marcas no tendrán más opción que desarrollarse en este campo.

Finalmente, otro aspecto relevante es que para las agencias de comunicación el tema de la contratación del recurso humano es un gran reto ya que la industria está migrando a valoraciones de habilidades relacionadas con el desarrollo interpersonal, diferentes backgrounds no necesariamente vinculados con el área de comunicación.

Por tratarse de un estudio exploratorio, se espera que los hallazgos estimulen la discusión en la industria y en la comunidad académica y que esto resulte en la generación de futuras investigaciones de mayor profundidad.

\section{Referencias}

Eiserman, Rick; Bond, Declan; Fraga, Amanda; Daryan, Sadie y Shreffler, Melanie. (2017, setiembre). "Brands: the new icons of culture". Ponencia presentada en la 14va Semana de la Publicidad, Advertising Week 2017, Nueva York. Recuperado de http://newyork.advertisingweek.com/ replay/-millennial-minded-seminar-2017-09-25-1330

García Avilés, José Alberto. (2009). La comunicación ante la convergencia digital: algunas fortalezas y debilidades. Signo y pensamiento, 28(54).

Fofstetter, Sarah; Keith, Kevin; Reardon, Sean y Sabol, Allison. (2017, setiembre). "Leadership and the agency of the future". Ponencia presentada en la 14va Semana de la Publicidad, Advertising Week 2017, Nueva York. Recuperado de http://newyork.advertisingweek.com/ replay/-4as-seminar-2017-09-25-1030

Lanas, Patricia Corredor. (2011). Nuevos profesionales en Publicidad. TELOS 87: Nuevos perfiles profesionales para la comunicación digital, 87, 97.

Lebendiker, Marcelo; Herrera, Rafael y Velázquez, Guillermo. (2015). "Reporte Nacional 2014: la situación del emprendimiento en Costa Rica". Global Entrepreneurship Monitor. 1er Ed. Recuperado de http://www.gemconsortium.org/report/49174

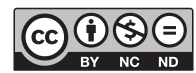

Rev. Reflexiones 98 (1) Enero-Junio, 2019: 39-60, E-ISSN: 1659-2859 
Martínez, Inmaculada; Aguado, Juan Miguel. (2014). Publicidad móvil: impacto presente y futuro en el ecosistema del contenido digital. Revista de la Asociación Española de Investigación de la Comunicación, 1(1), 76-85.

Porter, Michael. (2007). La ventaja competitiva de las naciones. Harvard Business Review América Latina. Vol 85, 11. 69-95.

Porter, Michael. (2017). Entrevista. Recuperado el 17 de octubre de https://www.youtube.com/ watch?v=Knwk15Q2OJ4

Sandoval, Carlos. (1996). Una aproximación al campo profesional de la publicidad en Costa Rica. Reflexiones. Vol 49, 1. 13-14.

Sandoval, Carlos y Al-Ghassani, Anwar. (1990). Inventario de los medios de comunicación en Costa Rica. San José (Costa Rica): Escuela de Ciencias de la Comunicación Colectiva, Universidad de Costa Rica.

Wacksman, Barry; Law, Nick y Radia, Saneel. (2017, setiembre). "Self-Disrupt: Creating the next agency model". Ponencia presentada en la 14va Semana de la Publicidad, Advertising Week 2017, Nueva York.Recuperadodehttp://newyork.advertisingweek.com/calendar/-rga-seminar-2017-09-25-1515

Weed, Keith. (setiembre, 2017). "The Future of marketing. Driving growth in a digital world”. Ponencia presentada en la 14va Semana de la Publicidad, Advertising Week 2017, Nueva York. Recuperado de http://newyork.advertisingweek.com/replay/-unilever-seminar-2017-09-25-1215 